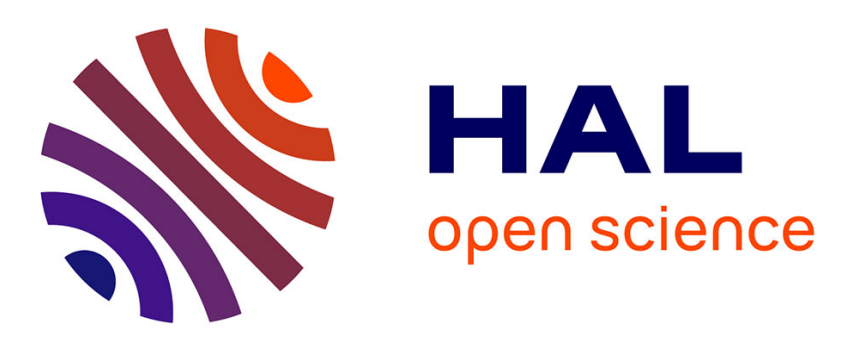

\title{
Giant Enhancement of Luminescence Down-Shifting by a Doubly Resonant Rare-Earth-Doped Photonic Metastructure
}

Ngoc-Vu Hoang, Antonio Pereira, Hai Son Nguyen, Emmanuel Drouard, Bernard Moine, Thierry Deschamps, Régis Orobtchouk, Anne Pillonnet, Christian Seassal

\section{To cite this version:}

Ngoc-Vu Hoang, Antonio Pereira, Hai Son Nguyen, Emmanuel Drouard, Bernard Moine, et al.. Giant Enhancement of Luminescence Down-Shifting by a Doubly Resonant Rare-Earth-Doped Photonic Metastructure. ACS photonics, 2017, 4 (7), pp.1705 - 1712. 10.1021/acsphotonics.7b00177 . hal01939297

\section{HAL Id: hal-01939297 https://hal.science/hal-01939297}

Submitted on 30 Nov 2018

HAL is a multi-disciplinary open access archive for the deposit and dissemination of scientific research documents, whether they are published or not. The documents may come from teaching and research institutions in France or abroad, or from public or private research centers.
L'archive ouverte pluridisciplinaire $\mathbf{H A L}$, est destinée au dépôt et à la diffusion de documents scientifiques de niveau recherche, publiés ou non, émanant des établissements d'enseignement et de recherche français ou étrangers, des laboratoires publics ou privés. 


\section{Giant enhancement of luminescence down-shifting by a}

\section{doubly-resonant rare-earth-doped photonic metastructure}

Ngoc-Vu Hoang, $,^{\dagger}, *$ Antonio Pereira, ${ }^{\dagger}, *$ Hai Son Nguyen, ${ }^{\dagger}$ Emmanuel Drouard, ${ }^{\ddagger}$ Bernard Moine, ${ }^{\dagger}$ Thierry Deschamps, ${ }^{\ddagger}$ Regis Orobtchouk, ${ }^{\S}$ Anne Pillonnet, ${ }^{\dagger}$ Christian Seassal ${ }^{+}, *$

†Univ Lyon, Université Claude Bernard Lyon 1, CNRS, Institut Lumière Matière, F-69622, Villeurbanne, France.

$\$$ Univ Lyon, Institut des Nanotechnologies de Lyon - INL, UMR CNRS 5270, CNRS, Ecole Centrale de Lyon, Ecully F-69134, France.

${ }^{\S}$ Univ Lyon, Institut des Nanotechnologies de Lyon - INL, UMR CNRS 5270, CNRS, INSA de Lyon, Villeurbanne F-69621, France.

\section{KEYWORDS}

Luminescence enhancement; rare earth; down-shifting; photonic crystal; metastructure

\section{ABSTRACT}

Phosphors based on rare earth ions are used to convert photons to useful wavelengths for a wide range of applications, but the improvement of their emission efficiency remains a key challenge in thin-films. In this paper, we demonstrate theoretically and experimentally that a photonic crystal (PC) structure can efficiently enhance the luminescence properties of a down-shifting layer (DSL). Our new metastructure combines a DSL $\left(\mathrm{Y}_{2} \mathrm{O}_{3}: \mathrm{Eu}^{3+}\right)$ and a two dimensional (2D) planar PC $\left(\mathrm{SiN}_{\mathrm{x}}\right)$, the latter being specifically engineered to control simultaneously the absorption in the DSL and the emission into free space. A global 
emission enhancement of 77 times is obtained due to PC Bloch mode resonances. Additionally, this metastructure exhibits high transmission above $450 \mathrm{~nm}$, and favours the emission of converted light at specific angles, with an enhancement factor of up to 690. We explain how the slow and fast Bloch modes of the PC structure induce these giant absorption and extraction enhancements of light. 
Phosphors based on rare earth (RE) ions play an increasingly important role in a variety of applications such as bio-imaging and labeling, ${ }^{1}$ displays, ${ }^{2}$ lighting, ${ }^{3}$ solar cells, ${ }^{4-7}$ thermometry, ${ }^{8}$ and X-ray imaging. ${ }^{9}$ Interest in these materials is related to their luminescent properties over a wide spectral range from ultraviolet (UV) to infra-red (IR), their high quantum efficiencies close to the theoretical maximum (100\%), and their long emission lifetimes (up to a few milliseconds). Unfortunately, due to the forbidden nature of the $4 \mathrm{f}-4 \mathrm{f}$ transitions in trivalent rare earth ions, such materials suffer from a small absorption crosssection (around $10^{-20} \mathrm{~cm}^{2}$ ), ${ }^{10}$ making them unsuitable for thin film applications for example. In order to improve the photoluminescence (PL) efficiency, several approaches can be considered. The simplest one consists of using a large amount of material (up to several tens of microns thick), but this is not compatible with thin film applications. Another possibility is to increase the doping concentration. However, the efficacy of this approach is limited because of emission quenching process. During the last few years, the maturity of nanophotonics, ${ }^{11}$ together with the mastering of nanofabrication, has enabled novel solutions to tailor light-matter interaction at the subwavelength scale. These technologies can now be used to improve the efficiency of light absorption, emission, or extraction of RE doped thin films. Thanks to plasmonic resonances, metallic structures precisely designed on the nanometer scale can give rise to a fluorescence enhancement in nearby emitters via processes occurring at the excitation and/or the emission wavelengths. By exploiting these properties, many studies have been devoted to plasmonicbased solutions using a handful of materials such as gold ${ }^{12-15}$, silver ${ }^{11-13,16}$ and aluminum. ${ }^{17,18}$ However, in practice, and summarizing the current state of the art, the fluorescence enhancement obtained with such coupling is limited to factors of a few tens, ${ }^{10,12-15}$ the highest plasmon mediated enhancement being achieved when the resonance is tuned in the excitation wavelength range. ${ }^{12}$ Recently, Lee et al. ${ }^{19}$ have reported improvements up to 29- and 174 times for down-shifted luminescence and up-shifted luminescence, respectively. However, due the complexity of the structures involved which combine simultaneously down-shifting and up-conversion layers with silver film and nanodisks, the origin of the 
enhancement factors is not fully understood. On the other hand, PC structures allow a careful engineering of the dispersion of photonic bands, and thereby make it possible to enhance both light extraction ${ }^{20}$ and absorption. ${ }^{21}$ Additionally, as these structure enable in-depth modification of the local density of optical states (LDOS), they may be used to control emission rates, ${ }^{22}$ and energy transfer mechanisms. ${ }^{23}$ These photonic approaches provide unique ways to improve the wavelength conversion in RE doped layers. Most of the literature in this field is focused on enhancing the RE-emission extraction, by out-coupling guided light via Bloch mode resonances of a PC, which leads to an increase in luminescence by a factor of several times. ${ }^{24-26}$ In a previous study using a $\mathrm{Ce}^{3+}, \mathrm{Pr}^{3+}, \mathrm{Yb}^{3+}$ tri-doped $\mathrm{CaYAlO}_{4}$ layer, we theoretically demonstrated that Bloch mode resonances at excitation wavelengths can lead to a 10-fold increase of the UV light absorption. ${ }^{27}$ Most recently, Hofmann et al. have proposed a one dimensional (1D) PC design which combines absorption improvement and LDOS enhancement, yielding a 480-fold theoretical up-conversion enhancement of $\mathrm{Er}^{3+}$ doped $\mathrm{NaYF}_{4}{ }^{28}$ Depending on the geometrical parameters of the photonic structure, it is therefore possible to optimize separately or simultaneously the RE ions absorption, emission rate and light extraction of the converting layer.

In this article, we present a new method to improve the down-shifting efficiency of light by combining a 2D planar PC slab with a luminescent DSL, as shown in Figure 1a. We choose the well-known phosphor $\mathrm{Y}_{2} \mathrm{O}_{3}: \mathrm{Eu}^{3+}$, now widely used in fluorescence lamps and field emission displays, ${ }^{29-31}$ as the DSL material. $\mathrm{The}^{\mathrm{Eu}} \mathrm{u}^{3+}$ ion is also well known for its intense red emission under excitation in the UV range, and for its quantum yield close to $100 \%$. A detailed study on the spectroscopic properties of $\mathrm{Eu}^{3+}$ ions can be found elsewhere. ${ }^{32}$ For the PC layer located on the top of the DSL, a highly transparent and high optical refractive index material, namely $\mathrm{SiN}_{\mathrm{x}}$, is used. As the luminescent down-shifting process involves an interplay between absorption, emission and extraction mechanisms, various strategies can be envisaged to enhance its efficiency. In this study, the PC structure is primarily designed in such a way as to enhance the absorption in the DSL. To reach this objective, the PC membrane should exhibit low Bloch modes at 
photonic band edges with almost-zero group velocity. We use the resonant coupling of these modes with the incident light to trap incoming photons within the PC structure and thus improve the absorption. ${ }^{21}$ In order to specifically enhance the absorption in the DSL, the PC dispersion must therefore be engineered to exhibit Bloch resonances spatially and spectrally tuned to the absorbing medium. These photonic structures should also be chosen to exhibit dispersion characteristics that favour light emission and extraction in the longer wavelength range.

\section{RESULTS AND DISCUSSION}

In this work, the geometrical parameters of the PC have been carefully chosen to optimize the absorption of incident light in a $100-\mathrm{nm}$ thick $\mathrm{Y}_{2} \mathrm{O}_{3}: \mathrm{Eu}^{3+}$ layer, and considering the specific case of the solar spectrum as the incident light. In our design, a square symmetry of air holes was chosen. The PC structural parameters, including the $\mathrm{SiN}_{\mathrm{x}}$ thickness $\mathrm{t}$, the lattice period $\mathrm{p}$, and the aspect ratio $\mathrm{D} / \mathrm{p}$ (D being the holes diameter), were varied to ensure high absorption in the DSL. The influence of the PC structural parameters on the absorption of the DSL is summarized with the figure of merit (FOM), which is calculated as

$$
F O M=\int A(\lambda) \cdot P L E(\lambda) \cdot S(\lambda) \cdot d \lambda
$$

where $A(\lambda)$ is the absorption spectrum of the device which is numerically calculated by the Rigorous Coupled Wave Analysis method (RCWA, see Materials and Methods section for more details), $P L E(\lambda)$ is the experimental photoluminescence excitation spectrum of $\mathrm{Eu}^{3+}$ (see Figure $2 \mathrm{a}$ ), and $S(\lambda)$ is the AM 1.5G solar irradiance. According to this optimization, the set of parameters selected for the fabrication is: 100 -nm-thick SiNx square lattice PC with period $p$ of $250 \pm 6 \mathrm{~nm}$, holes diameter $D$ of $175 \pm 25 \mathrm{~nm}$ (i.e. an aspect ratio $\mathrm{D} / \mathrm{p}$ of $0.7 \pm 0.1$ ), and etching depth $h$ of $90 \pm 10 \mathrm{~nm}$. The FOM of our new downshifting structure was calculated by scanning the PC geometrical parameters and it is shown in Figure SI.1 in the Supporting Information. 

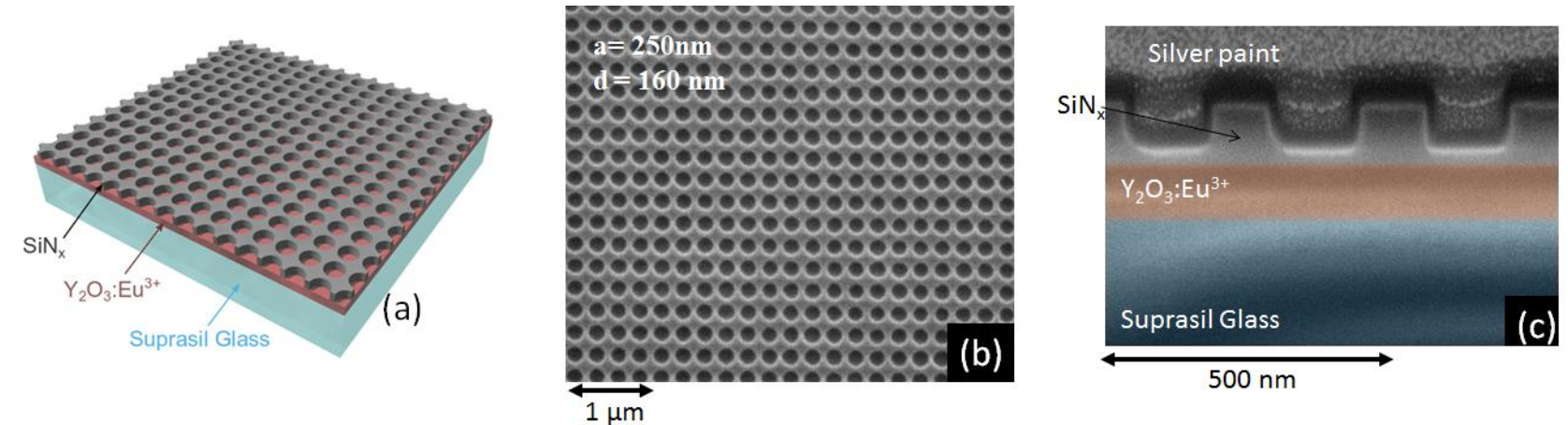

Figure 1. (a) Schematic view of the new down-shifting metastructure combining a 100-nm thick $\mathrm{Y}_{2} \mathrm{O}_{3}: \mathrm{Eu}^{3+}$ as the DSL and a 2D PC layer. (b) SEM top view of the 2D PC covering the $\mathrm{Y}_{2} \mathrm{O}_{3}$ :Eu ${ }^{3+}$ layer. (c) SEM cross-sectional view of the whole structure (the sample surface is covered with silver paint to avoid charging effects).

We used different nanofabrication steps to produce the designed photonic metastructure (see Materials and Methods section and Figure SI.2 in Supporting Information for more details). The DSL, i.e. a 100nm-thick $\mathrm{Y}_{2} \mathrm{O}_{3}: \mathrm{Eu}^{3+}$ film, was first deposited on Suprasil glass by means of pulsed laser deposition. Subsequent annealing under air at $800^{\circ} \mathrm{C}$ for $6 \mathrm{~h}$ was performed to improve its crystallization. Structural, morphological and optical properties of the annealed $\mathrm{Y}_{2} \mathrm{O}_{3}: \mathrm{Eu}^{3+}$ layer are given in the Supporting Information (Figures SI.3 and SI.4). The PC was then fabricated on top of the $\mathrm{Y}_{2} \mathrm{O}_{3}:$ Eu$^{3+}$ layer following three steps: (i) deposition of a 100-nm thick $\mathrm{SiN}_{\mathrm{x}}$ layer using low-frequency plasma enhanced chemical vapor deposition, (ii) laser interference lithography and (iii) reactive ion etching in order to transfer the pattern onto the $\mathrm{SiN}_{\mathrm{x}}$ layer. Scanning Electronic Microscope (SEM) images of the fabricated structure are shown in Figure 1b,c. These SEM views show the 2D PC of 250-nm period, covering the $\mathrm{Y}_{2} \mathrm{O}_{3}: \mathrm{Eu}^{3+}$ layer. Holes are $80 \mathrm{~nm}$ deep, with diameter $D$ of $160 \mathrm{~nm}$. The whole sample is $12.5 \mathrm{~mm}$ x $12.5 \mathrm{~mm}$ (see Figure $2 \mathrm{~b}$ ) with a highly uniform PC pattern ( $D$ varies by about $5 \%$ within $100 \mu \mathrm{m} \times 100 \mu \mathrm{m})$. The Gaussian distribution of the hole diameter, extracted from SEM images, is shown in the Supporting Information (Figure SI.6). 

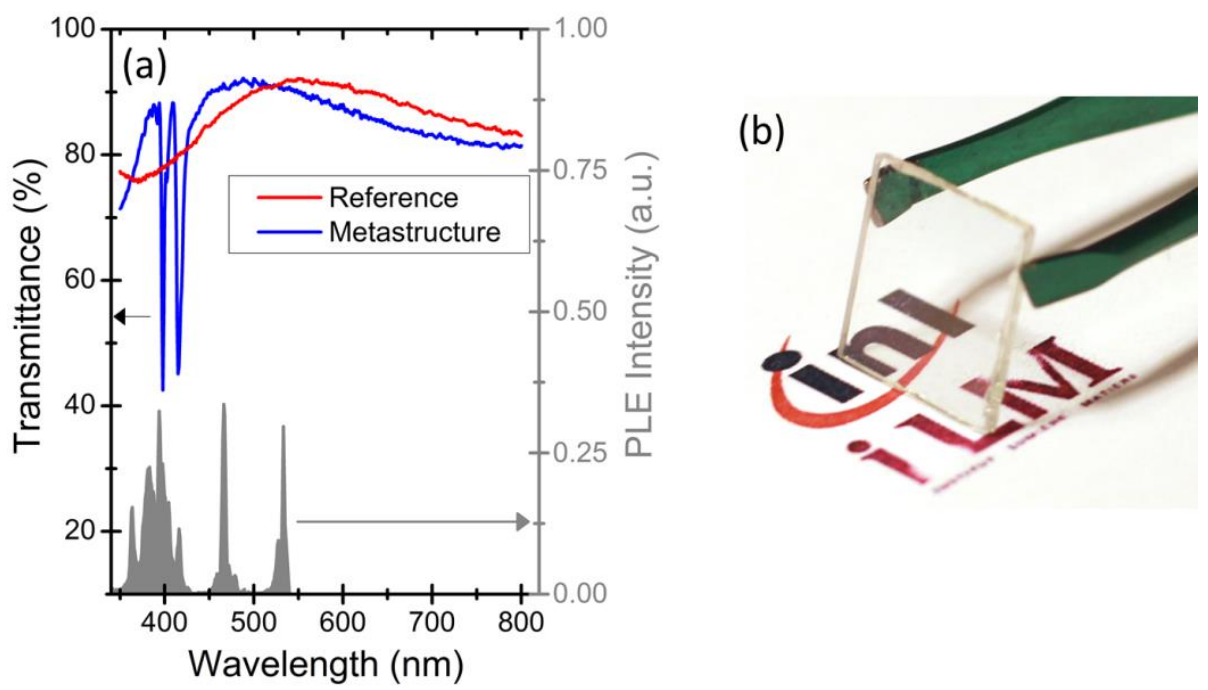

Figure 2. (a) Transmittance spectrum of the metastructure (sample with PC - blue curve) and of the reference (full stack with unpatterned $\mathrm{SiNx}$ - red curve). The photoluminescence excitation spectrum of $\mathrm{Eu}^{3+}$ is also given by the grey curve. (b) Picture of the whole sample combining the DSL and the 2D PC slab.

The effect of the PC structure on the transmission of the metastructure over the solar spectrum was first investigated. Transmittance spectra of the metastructure (sample with PC - blue curve) and of the reference (full stack with unpatterned $\mathrm{SiNx}$ - red curve) measured at normal incidence are shown in Figure 2a. These spectra clearly reveal that the PC structure generates two resonant modes in the DSL at 400 and $419 \mathrm{~nm}$. As expected, these two modes are perfectly coupled with the excitation bands of $\mathrm{Eu}^{3+}$ between 350 and $430 \mathrm{~nm}$ (grey curve). Another important result is that the transparency of the DSL is globally not affected by the presence of the $\mathrm{SiN}_{\mathrm{x}} \mathrm{PC}$. More precisely, the transmittance remains within $80 \%$ to $93 \%$ in the visible and near infrared domains. However, the maximum transmission is blueshifted, which can be attributed to a decrease of the effective refractive index due to the presence of the air holes in the structure. Figure $2 b$, showing a macroscopic image of the whole sample, illustrates this high transparency. 


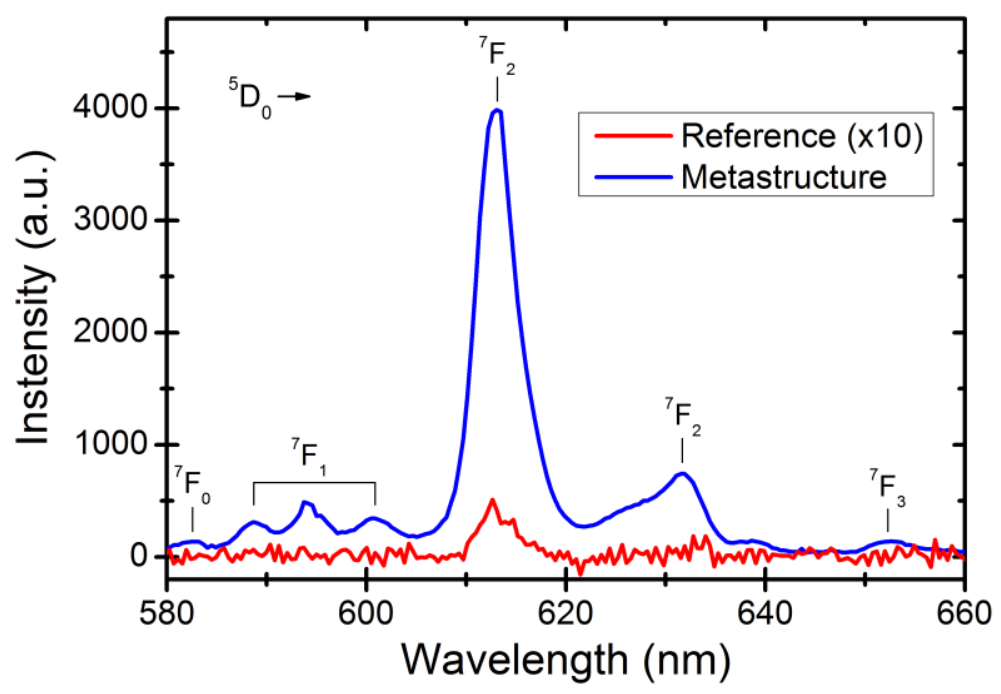

Figure 3. Luminescence properties of the metastructure (sample with PC - blue curve) and of the reference (full stack with unpatterned SiNx - red curve). The PL intensity of the reference sample is magnified 10 times to be distinguishable.

To demonstrate the performance of the proposed device, PL measurements were first performed in an integrating sphere with an optical excitation of $400 \mathrm{~nm}$ at normal incidence (with a power density of 0.82 $\mathrm{mW} / \mathrm{mm}^{2}$ and a spot size of $1 \mathrm{~mm}$ diameter, more details can be found in Materials and Methods section). The emission spectra of the metastructure and reference samples are shown in Figure 3. The observed emission spectra exhibit peaks corresponding to the transitions ${ }^{5} D_{0} \rightarrow{ }^{7} F_{J}$ (with $J=0-3$ ) of Eu ${ }^{3+}$ ions in the cubic phase. As expected, the $\mathrm{Y}_{2} \mathrm{O}_{3}: \mathrm{Eu}^{3+}$ film exhibits very low PL intensity when the SiN $\mathrm{x}_{\mathrm{x}}$ layer is unpatterned (red curve), and only the luminescence of the main transition ${ }^{5} D_{0} \rightarrow{ }^{7} F_{2}$ at $611 \mathrm{~nm}$ is observed. The light trapping effect is clearly evidenced on the patterned sample (blue curve). Indeed, thanks to the $\mathrm{SiN}_{\mathrm{x}} \mathrm{PC}$ a $77 \pm 3$ fold enhancement of the emission is measured and all the emission peaks corresponding to the transitions ${ }^{5} D_{0} \rightarrow{ }^{7} F_{J}$ (with $J=0-3$ ) are now clearly distinguishable. This PL enhancement is about 10 times higher than the best results reported in literature for DSLs such as $\mathrm{Y}_{2} \mathrm{O}_{3}: \mathrm{Eu}^{3+}$ thin films. ${ }^{24,33} \mathrm{We}$ will now analyse the contributions of the different mechanisms to this remarkable conversion increase, in both the absorption and emission wavelength ranges. 

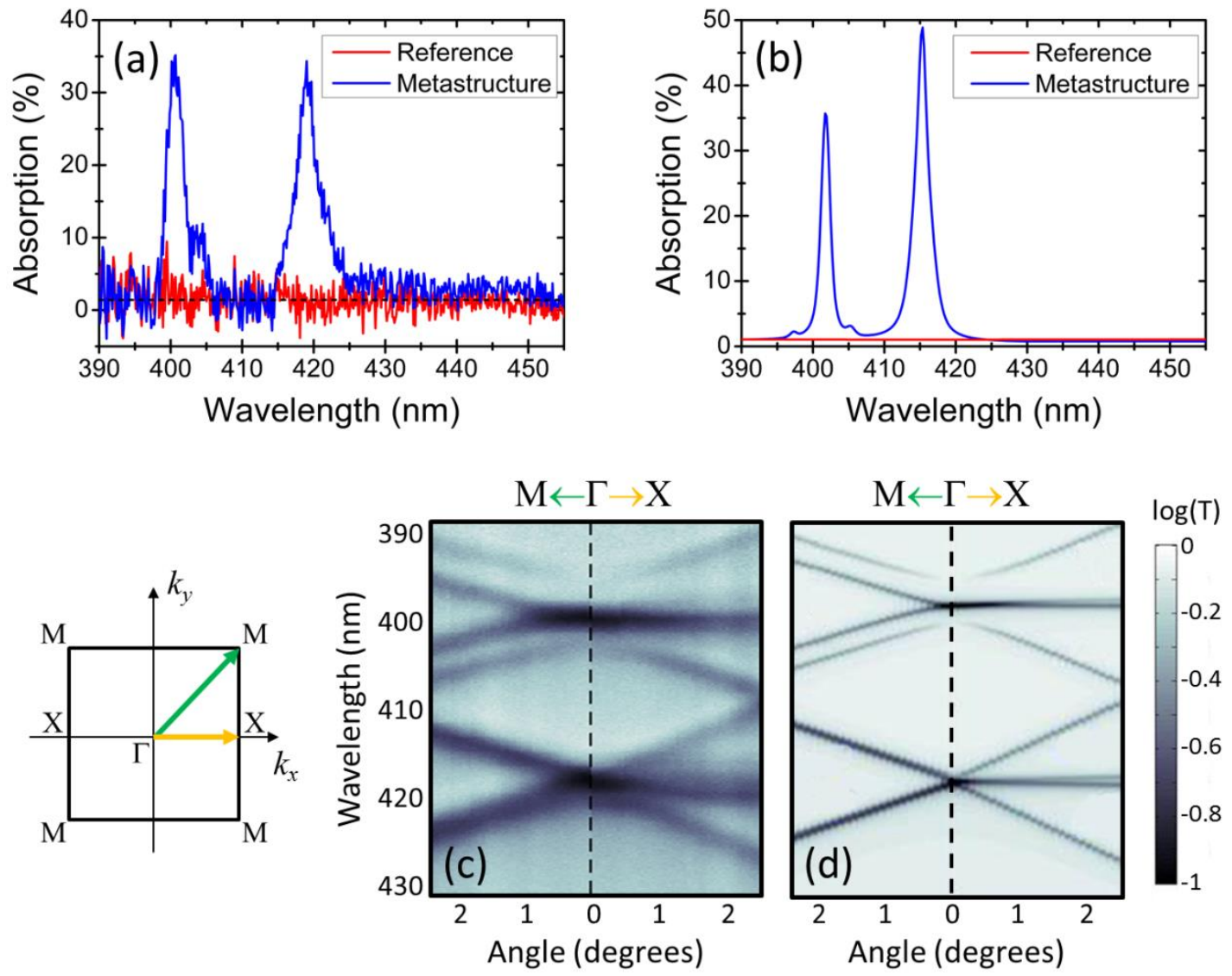

Figure 4. (a) Experimental measurements and (b) theoretical simulations of the absorption spectra at normal incidence of the metastructure (sample with PC - blue curve) and of the reference sample (full stack with unpatterned SiNx - red curve)). The dotted black line is the average reference absorption. (c) Experimental measurements and (d) theoretical simulations of the angularly resolved transmission of the metastructure.

Figure 4a depicts absorption spectra of the metastructure and reference samples. The absorption was extracted from transmission and reflectivity spectra measured at normal incidence (the excitation source was a halogen lamp and the diameter of the excitation spot was $100 \mu \mathrm{m}$ with angular aperture of $0.2^{\circ}$ ). The spectrum of the metastructure exhibits two clear and intense absorption peaks, corresponding to resonances at $400 \mathrm{~nm}$ and $419 \mathrm{~nm}$. We note that the measured data includes the global absorption of Eu ${ }^{3+}$ in the $\mathrm{Y}_{2} \mathrm{O}_{3}$ host matrix (the parasitic absorption of $\mathrm{SiN}_{\mathrm{x}}$ and Suprasil glass are negligible). Since Eu ${ }^{3+}$ and $\mathrm{Y}_{2} \mathrm{O}_{3}$ are located in the same layer with respect to the electric field distribution, they share the same 
enhancement factor of absorption. From the measurements, the absorption enhancement factor at $400 \mathrm{~nm}$ is given by

$$
\eta_{a b s}=\frac{A_{400 \mathrm{~nm}}^{M e t}}{A_{400 \mathrm{~nm}}^{R e f}}=25 \pm 5
$$

where $A_{400 \mathrm{~nm}}^{\mathrm{Met}}$ and $A_{400 \mathrm{~nm}}^{R e f}$ are the absorption of the metastructure and reference samples, respectively. The simulated spectrum as calculated by RCWA is displayed in Figure $4 \mathrm{~b}$ (see Materials and Methods section for more details). The simulations, which take into account the 5\% variation in hole diameter on the fabricated sample and the angular aperture of the excitation beam, are in good agreement with the observed absorption spectrum. The slight shift in spectral position with respect to the experimental measurements is attributed to the imperfect shape of the PC holes. In particular, they are incompletely etched truncated cones instead of perfect cylinders (Figure 1c). To further characterize the two absorption resonances, we performed angularly-resolved transmission measurements around the normal incidence along the $\Gamma$-X and $\Gamma$-M symmetry axes (see Materials and Methods section for more details). The experimental spectrum (Figure 4c) is in very good agreement with predictions provided by the RCWA simulation (Figure 4d and Figure SI.7 in Supporting Information). Both spectra clearly show a photonic dispersion corresponding to slow Bloch modes at $400 \mathrm{~nm}$ and $419 \mathrm{~nm}$. This result confirms the fact that the slow Bloch mode at $400 \mathrm{~nm}$ is responsible for the absorption enhancement.

Angularly resolved PL experiments were also performed on the reference (Figure 5a) and on the metastructure (Figure 5b) under excitation at $400 \mathrm{~nm}$ and at normal incidence (with a power density of $0.82 \mathrm{~mW} / \mathrm{mm}^{2}$ and a spot size of $1 \mathrm{~mm}$ diameter). The PL was measured under a conic angle $\phi=0^{\circ}$ for various emission angles $\theta$ ranging from 10 to $170^{\circ}$. The angular distribution of the light emission corresponding to the main transition ${ }^{5} D_{0} \rightarrow{ }^{7} F_{2}$ is shown in Figure 5c. The corresponding configuration is shown in Figure 5d. As expected, the emission pattern of the reference (Figure 5a) does not exhibit an optical dispersion characteristic; light emitted around $611 \mathrm{~nm}$ is extracted directly into free-space over a 
large angular range. On the contrary, the emission pattern of the metastructure (Figure 5b) clearly exhibits two photonic bands corresponding to Bloch resonances in a background of free-space modes. Thus, the metastructure preferentially extracts emitted photons at angles selected by the Bloch mode dispersion characteristics, and they overlap with the main transition of the emitter at $611 \mathrm{~nm}$ (Supporting Information for details, Figures SI.8 and SI.9).
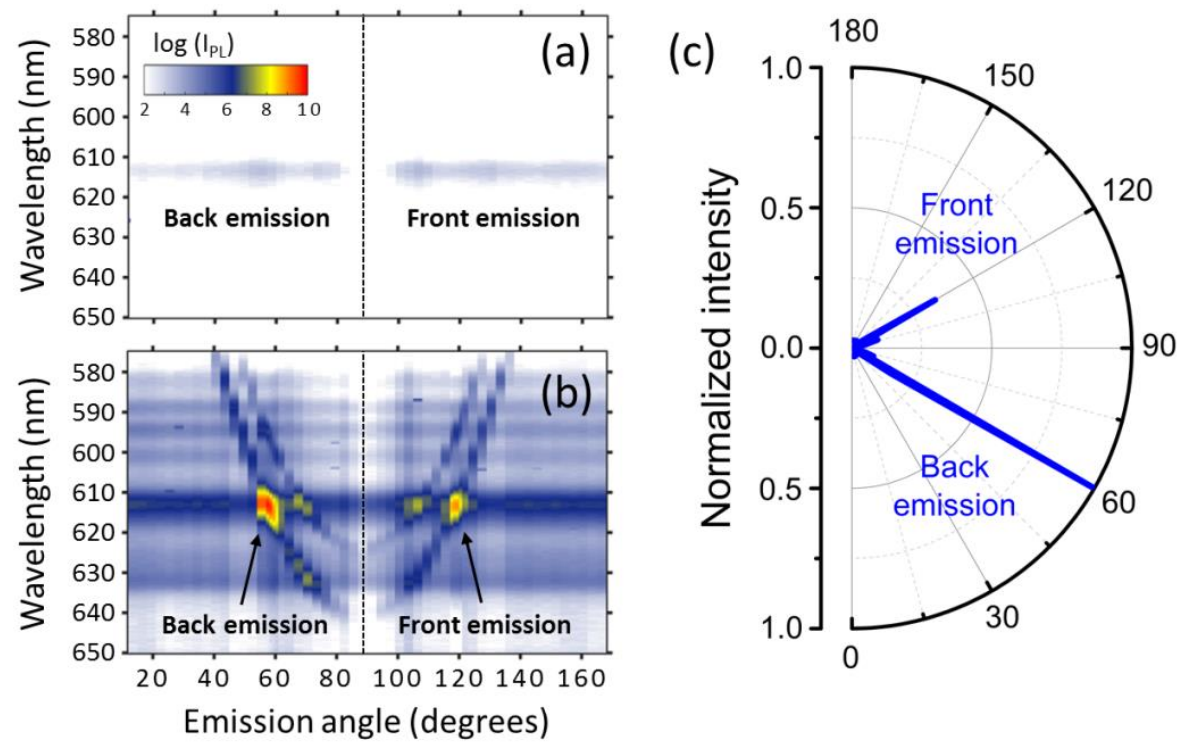

(d)

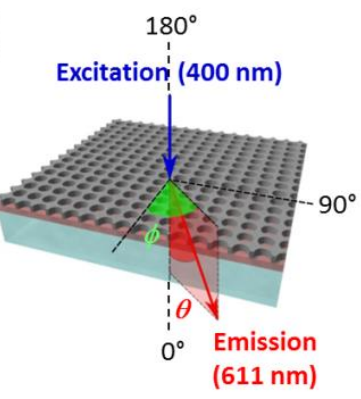

Figure 5. Angularly-resolved PL experiments on the reference (a) and on the metastructure sample (b) under excitation at $400 \mathrm{~nm}$ (the data are presented in log-scale to highlight Bloch modes at different energies since the signal at $611 \mathrm{~nm}$ is too dominant on a linear scale). (c) Angular distribution of emission at $611 \mathrm{~nm}$ (corresponding to the main transition ${ }^{5} D_{0} \rightarrow{ }^{7} F_{2}$ ). (d) Schematic of the angularly resolved PL experiment.

These results clearly show that our metastructure not only controls the absorption at $400 \mathrm{~nm}$ with slow Bloch modes, but also influences the extraction in the $\mathrm{Eu}^{3+}$ emission range due to fast Bloch modes, i.e. with a significant slope leading to a high group velocity. Such dispersions provide efficient out-coupling channels via Bloch resonances, and thus enhance extraction of emission. ${ }^{34}$ The angular distribution of emission at $611 \mathrm{~nm}$, depicted in Figure 5c, shows a high degree of anisotropy - mostly unidirectional with an oblique emission angle: $\theta=58^{\circ}$ (back emission) and $\theta=119^{\circ}$ (front emission). In addition to 
that, the metastructure favours the transmitted emission since the back emission, i.e. below the metastructure, into the substrate, is about twice as intense as the front emission (Figure 5c,d). We attribute this property to the vertical asymmetry of the stack, which includes a higher refractive index medium below the PC structure compared to the ambient medium above it.

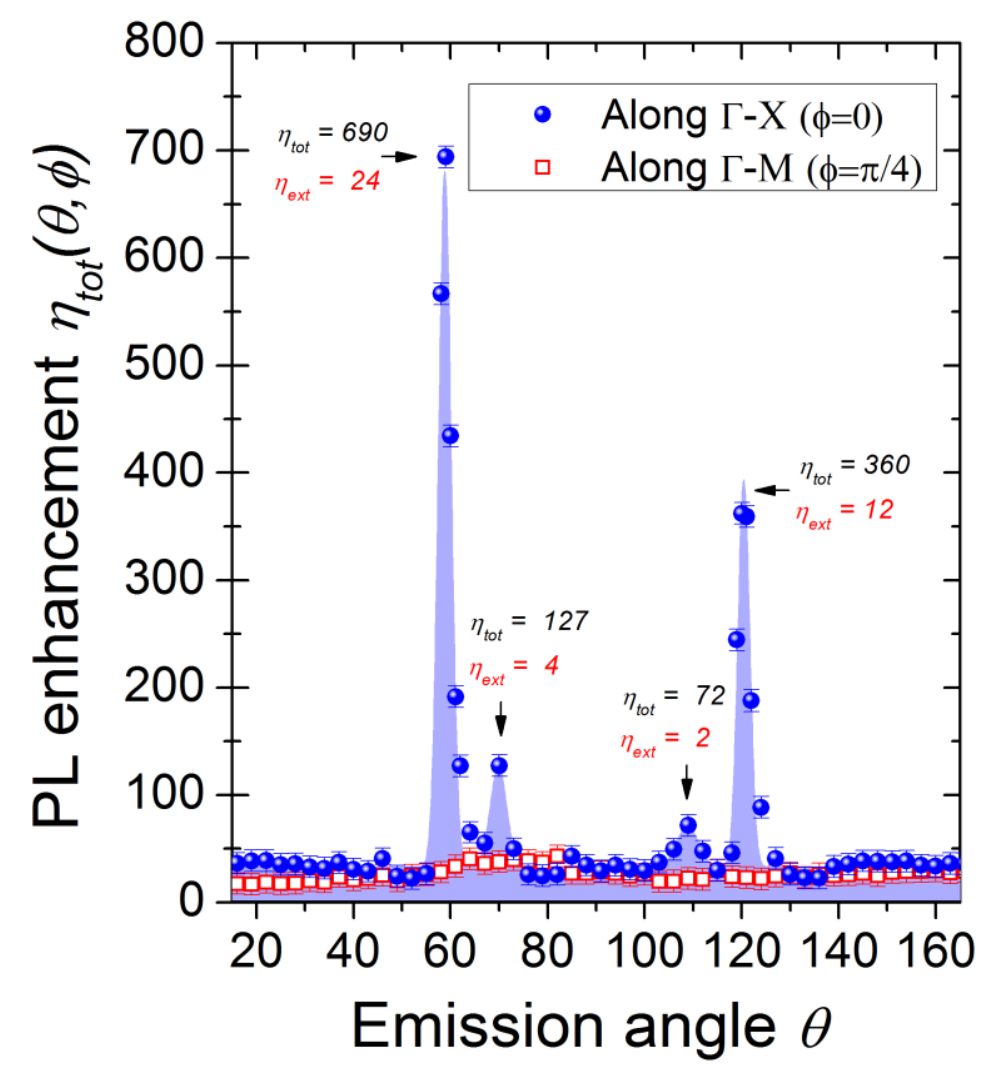

Figure 6. Total PL enhancement $\eta_{t o t}(\theta, \phi)$ extracted from the angularly-resolved PL measurements for the incident plans $\Gamma$-X (i.e. $\phi=0$ ) and $\Gamma$-M (i.e. $\phi=\pi / 4$ ). The enhancement factor is given by $\eta_{\text {tot }}(\theta, \phi)=$ $\eta_{a b s} \cdot \eta_{\text {ext }}(\theta, \phi) . \eta_{\tau}$, where $\eta_{a b s}$ is the absorption enhancement factor due to slow Bloch modes at 400 $\mathrm{nm}, \eta_{\text {ext }}(\theta, \phi)$ is the extraction enhancement factor due to fast Bloch modes at $611 \mathrm{~nm}$ and $\eta_{\tau}$ is the emission rate enhancement factor. The values given in the figure correspond to the $\Gamma$-X direction.

Figure 6 presents the total PL enhancement $\eta_{t o t}(\theta, \phi)$ extracted from the angularly resolved PL measurements performed on the metastructure and on the reference. This total enhancement $\eta_{\text {tot }}(\theta, \phi)$, 
which is the average of the enhancement factor with emission angle $\theta \in[0, \pi]$, and emission plan making an angle $\phi \in[0,2 \pi]$ with the PC axis (see Figure $5 \mathrm{~d}$ ) is given by

$$
\eta_{\text {tot }}(\theta, \phi)=\frac{1}{4 \pi} \iint \eta(\theta, \phi) \sin \theta \cdot d \theta \cdot d \phi \text {. }
$$

The measurements were therefore performed according to both planes of incidence $\Gamma$-X (i.e. $\phi=0$ ) and $\Gamma$-M (i.e. $\phi=\pi / 4$ ) to determine how the emission is enhanced in different directions and thereby to infer the total enhancement. In the case of the $\Gamma$-M direction (red dots), the enhancement is almost constant and equal to $\eta_{t o t} \sim 26 \pm 7$ times for all angles. Such enhancement is consistent with the slow Bloch mode observed previously at $400 \mathrm{~nm}$ following the $\Gamma$-M direction, and is responsible for the absorption enhancement (Figure $4 \mathrm{c}$ ). In the case of the $\Gamma$-X direction (blue dots), a similar trend is observed, namely a constant background enhancement of $\eta_{t o t} \sim 33 \pm 7$, except for four highly intense emission angles located at $59^{\circ}, 70^{\circ}, 109^{\circ}$ and $120^{\circ}$. These are attributed to preferential out-coupling to fast Bloch modes displayed in Figure 5b. Moreover, we highlight that the highest global wavelength conversion enhancement is obtained at $\theta=59^{\circ}$, with $\eta_{\text {total }} \sim 690$. These results show a strong angular dependence of the enhanced emission, suggesting that the increased absorption is not the only mechanism responsible for such luminescence enhancement. Indeed, for each $(\theta, \phi)$, the enhancement factor is also given by

$$
\eta_{t o t}(\theta, \phi)=\eta_{a b s} \cdot \eta_{e x t}(\theta, \phi) . \eta_{\tau}
$$

where $\eta_{a b s}$ is the absorption enhancement factor due to slow Bloch modes at $400 \mathrm{~nm}$ (see Figure 4), $\eta_{\text {ext }}(\theta, \phi)$ is the extraction enhancement factor due to fast Bloch modes at $611 \mathrm{~nm}$ (see Figure 5) and $\eta_{\tau}$ the emission rate enhancement factor of the metastructure with respect to the reference. Decay time measurements (Supplemental Information, Figure SI.10) show that the luminescence lifetime of $\mathrm{Eu}^{3+}$ does not change when the $\mathrm{SiN}_{\mathrm{x}}$ structure is patterned as a $\mathrm{PC}(\sim 1.1 \mathrm{~ms})$, indicating that the Purcell effect is not a relevant mechanism in our case (i.e. $\eta_{\tau}=1$ ). In summary, the observed emission 
enhancement is related to an improvement in the absorption efficiency, extraction efficiency, or a combination of both. A detailed analysis of Figures 4 and 6 reveals that, when there is no fast mode at play, i.e. in the $\Gamma$-M direction and for most angles in the $\Gamma$-X direction (except in the case of the intense emission peaks), the total enhancement arises only from an increase in the absorption. In these case, the absorption is on average enhanced 29 -fold (the variations observed are within experimental uncertainties), and fast Bloch modes do not enhance light extraction (i.e. $\eta_{\text {ext }}=1$ ). Using the same approach, we can therefore determine the extraction enhancement factor for each highly intense emission angles in the $\Gamma$-X direction. For example at $\theta=59^{\circ}$, with $\eta_{t o t} \sim 690$ and $\eta_{a b s} \sim 29$, we found that the extraction was enhanced by a factor of $\sim 24$. It is worth noting that this giant enhancement occurs only in one direction (i.e. an oblique emission which is favoured in the transmitted direction). This is an important property which can open new avenues for the implementation of DSL in photovoltaic applications. Indeed, such obliquely emitted light is expected to be efficiently trapped in a solar cell that could be positioned underneath the metastructure. Integrating these metastructures with silicon solar cell is currently under investigation. More generally, using this concept, tight adjustments of the geometrical parameters of the PC will enable one to tune the wavelength and emission angle of the converted light.

\section{CONCLUSION}

In summary, we have designed and fabricated a new metastructure that combines a luminescent downshifting layer with a photonic crystal, in order to control and enhance the down-shifting process. We have experimentally demonstrated efficiency enhancement of luminescence of the active layer using optical resonances of the PC structure. Our metastructure results in a 77 fold-enhancement of luminescent downshifting efficiency, which is the highest reported PL enhancement for DSLs such as $\mathrm{Y}_{2} \mathrm{O}_{3}: \mathrm{Eu}^{3+}$ thin films. Optical simulations and measurements demonstrate that the fabricated metastructure based on our design leads to slow Bloch resonances spectrally tuned with the absorption peaks of the DSL in the UV range. This coupling allows enhancement of the absorption in the DSL by up to 29 times without disturbing the 
transmission at other wavelengths. Due to fast Bloch modes in resonance with emission of $\mathrm{Eu}^{3+}$ at 611 $\mathrm{nm}$, which strongly improve the extraction process, giant luminescence enhancement can be achieved, up to a factor of 690 at specific angles in the transmitted direction. Such oblique emission is an important property which can open the door for the implementation of DSL in photovoltaic applications. One can expect a boost in efficiency if effective solar spectrum harvesting is realized, especially in the UV and near-UV range. More generally, this light management approach, combining DSLs and PCs, is relevant to improve the PL efficiency of other devices, such as LEDs and photodetectors where efficiency and directionality of the emitted light needs to be controlled. Specific metastructures can then be designed using our approach, and fabricated over wide areas using laser interference lithography or nanoimprinting, which is expected to open new routes for the implementation of a wide variety of emitting devices based on luminescent materials.

\section{MATERIALS AND METHODS}

Optical simulations. All the optical simulations use RCWA ${ }^{35}$ method. In our RCWA code developed in-house, analytical Bessel functions are used to calculate the Fourier coefficients of the permittivity for each cylindrical hole, rather than the Fast Fourier transform (FFT) techniques. This enables a faster computation. Moreover, in contrast to most other methods, the computation time does not depend on the thickness of the stack for a given spectral resolution. The number of orders used in the simulation, from -5 to +5 in each direction for the square lattice of holes, is sufficient to ensure accurate results. The absorption and angularly resolved transmission of the metastructure are simulated using a period of 250 $\mathrm{nm}$, an etching depth of $80 \mathrm{~nm}$, and the radius distribution shown in Figure SI.6.

Metastructure fabrication. $\mathrm{Y}_{2} \mathrm{O}_{3}: \mathrm{Eu}^{3+}(10$ at.\%) was first deposited by pulsed laser deposition using an ArF excimer laser beam $(\lambda=193 \mathrm{~nm}, \tau=17 \mathrm{~ns}$, Coherent CompexPro) operating at $5 \mathrm{~Hz}$. The $\mathrm{Y}_{2} \mathrm{O}_{3}: \mathrm{Eu}^{3+}$ target was previously synthesized by solid-state reaction $\left(8 \mathrm{~h}\right.$ at $1400{ }^{\circ} \mathrm{C}$ in air) after grinding 
and mixing $\mathrm{Y}_{2} \mathrm{O}_{3}$ (99.99\% purity, Alfa Aesar) and $\mathrm{Eu}_{2} \mathrm{O}_{3}$ (99.999\% purity, Alfa Aesar). Thin films were grown at room temperature in an oxygen-gas atmosphere $\left(\mathrm{P}_{\mathrm{O} 2}=10^{-3} \mathrm{mbar}\right)$. The laser fluence was held constant $3.2 \mathrm{~J} / \mathrm{cm}^{2}$. Depositions were performed on high purity fused silica glass (Suprasil grade, Heraeus), which was placed parallel to the target surface at a target-to-substrate distance of $5 \mathrm{~cm}$. The number of laser pulses was adjusted to achieve a $100 \mathrm{~nm}$ film thickness. The deposition rate (in nm/pulse) was first determined by means of a profilometer (Tencor Alpha Step 100). The deposited films were then subject to thermal annealing under air at $800{ }^{\circ} \mathrm{C}$ at a rate of $0.5^{\circ} / \mathrm{min}$ for $6 \mathrm{~h}$, and then cooled down to $20{ }^{\circ} \mathrm{C}$ at a rate of $0.5^{\circ} \mathrm{C} / \mathrm{min}$. Such thermal treatment allows for improvement of the crystallization of $\mathrm{Y}_{2} \mathrm{O}_{3}$, and the low ramping rate was used to avoid the formation of cracks on the film surface.

A 100 -nm thick $\mathrm{SiN}_{\mathrm{x}}$ layer was deposited on top of the $\mathrm{Y}_{2} \mathrm{O}_{3}$ : $\mathrm{Eu}^{3+}$ layer by low-frequency plasmaenhanced chemical vapor deposition, using a $440 \mathrm{kHz}$ SEMCO-Engineering reactor. The $\operatorname{SiN}_{\mathrm{x}}$ stoichiometry was controlled by adjusting the $\mathrm{SiH}_{4} / \mathrm{NH}_{3}$ gas ratio. The optical properties of $\mathrm{SiN}_{\mathrm{x}}$ were determined by ellipsometry measurements to ensure a low extinction coefficient $\mathrm{k}\left(<10^{-6}\right.$ for $\lambda \geq 350$ $\mathrm{nm}$ ) and the proper refractive index $\mathrm{n}$ in the working spectrum (Supporting Information, Figure SI.5). Laser interference lithography (LIL) ${ }^{36}$ was used to create a square lattice of air holes in the SiNx layer. HMDS (hexamethyldisilizane - an adhesion promoter) and then Ma-N2403 photoresist were first spincoated on the layer at $6000 \mathrm{rpm}$ for $30 \mathrm{~s}$, followed by baking on a contact hotplate at $90{ }^{\circ} \mathrm{C}$ for $60 \mathrm{~s}$. Exposure was performed with a $266 \mathrm{~nm}$ laser $(\mathrm{P}=10 \mathrm{~mW})$ on a sample holder connected to a mirror at $90^{\circ}$. Under illumination fringes are generated on the resist with an inter-fringe period $\mathrm{p}$. This is directly related to the angle $\theta$, which corresponds to the angle between the laser beam and the normal of the sample surface $\left(\mathrm{p}=\lambda_{\text {laser }} /(2 \sin \theta)\right)$. The hole radius is controlled by the exposure time $(336 \mathrm{~s})$. After tilting the sample at $90^{\circ}$, a second exposure was performed in order to create a $2 \mathrm{D}$ pattern with square symmetry. Finally, the resin was developed using a MIF726 solution for 20 s. After LIL, a reactive ion etching process was used to transfer the pattern from the photoresist on to the $\mathrm{SiN}_{\mathrm{x}}$ layer. The etching was 
performed using a gas mixture of $\mathrm{SF}_{6}, \mathrm{Ar}$ and $\mathrm{CHF}_{3}$ (flow rate of 15, 10 and $40 \mathrm{sccm}$ respectively), a total pressure of $30 \mathrm{~m}$ Torr and $100 \mathrm{~W}$ of power. The etching time was set to $70 \mathrm{~s}$. At the end, an additional oxygen cleaning plasma was performed for $120 \mathrm{~s}$ to completely remove the photoresist (flow rate of 20 sccm, pressure of $20 \mathrm{mT}$ Torr and $60 \mathrm{~W}$ of power).

Photoluminescence measurements. PL enhancement measurements were performed using a tunable pulsed laser ( $\tau=5 \mathrm{~ns}$, Ekspla NT340) as the excitation source. The fluorescence was collected through an optical fiber $(1.1 \mathrm{~mm}$ diameter, $\mathrm{NA}=0.2)$ connected to an integrating sphere (Labsphere), and coupled with a Triax190 spectrometer (Jobin Yvon) equipped with a CCD camera. All measurements were performed inside the integrating sphere under normal incidence with a $400 \mathrm{~nm}$ excitation wavelength (with a power density of $0.82 \mathrm{~mW} / \mathrm{mm}^{2}$ and a spot size of $1 \mathrm{~mm}$ diameter), six accumulations and $10 \mathrm{~s}$ of integration time. Angularly-resolved PL experiments were performed outside the integrating sphere using the same experimental setup. All spectra were corrected by the response of the experimental setup.

Angularly resolved transmission measurements. Incident broad light (halogen lamp) was focused onto the metastructure via a microscope objective $(x 20, N A=0.42)$, corresponding to a $50 \mu \mathrm{m}$ spot size. The transmitted light was collected via a second microscope objective $(x 5, \mathrm{NA}=0.2)$ whose the back focal plan was projected onto the entrance slit of a spectrometer. Finally, the output of the spectrometer was coupled to the sensor of a CCD camera (1024 x 256). This experiment allowed to measure in single shot the angularly resolved transmission (within the numerical aperture of the collecting objective): the horizontal axis of the CCD sensor is the spectral axis and the vertical axis is the angular one.

\section{ASSOCIATED CONTENT}

\section{Supporting Information}

The Supporting Information is available free of charge on the ACS Publications website.

This includes detailed information on the design of the 2D photonic crystal (figure of Merit); details on the fabrication and characterization of the new metastructure; SEM image of the fabricated PC structure 
and calculation of the diameter distribution of the holes; theoretical calculation of the dispersion diagram of the Bloch modes of the metastructure; details on extraction enhancement for different in-plane directions; lifetime measurements performed on the metastructure and the reference sample.

\section{AUTHOR INFORMATION}

\section{Corresponding Authors}

*E-mail: ngoc-vu.hoang@univ-lyon1.fr

*E-mail: antonio.pereira@univ-lyon1.fr

*E-mail: christian.seassal@ec-lyon.fr

\section{Author contributions}

The manuscript was written with contributions from all authors. All authors have given approval to the final version of the manuscript.

\section{Notes}

The authors declare no competing financial interest.

\section{ACKNOWLEDGMENTS}

We thank Dr Thomas David for SEM-FIB measurements. This work was supported by ARC 4 - Energies programme of the Auvergne-Rhône-Alpes Region.

\section{REFERENCES}

1. Bouzigues, C.; Gacoin, T.; Alexandrou, A. Biological Applications of Rare-Earth Based Nanoparticles. ACS Nano 2011, 11, 8488-8505.

2. Downing, E.; Hesselink, L.; Ralston, J.; Macfarlane, R. A Three-Color, Solid-State, ThreeDimensional Display. Science 1996, 273, 1185-1189. 
3. Meyer, F.; Trappe, F. Photoluminescent Materials for Solid-State Lighting: State of the Art and Future Challenges. Adv. Opt. Mater. 2015, 3, 424-430.

4. Trupke, T.; Green, M.A.; Wurfel, P. Improving Solar Cell Efficiencies by Down-Conversion of High-Energy Photons. J. Appl. Phys. 2002, 92, 1668-1674.

5. Richards, B.S. Luminescent Layers for enhanced Silicon Solar Cell Performance: DownConversion. Sol. Energy Mater. Sol. Cells 2006, 90, 1189-1207.

6. Huang, X.; Han, S.; Huang, W.; Liu, X. Enhancing Solar Cell Efficiency: The Search for Luminescent Materials as Spectral Converters. Chem. Soc. Rev. 2013, 42, 173-201.

7. Goldschmidt, J. C.; Fisher, S. Upconversion for Photovoltaics - A Review of Materials, Devices and Concepts for Performance Enhancement. Adv. Opt. Mater. 2015, 3, 510-535.

8. Jaque, D.; Vetrone, F. Luminescence Nanothermometry. Nanoscale 2012, 4, 4301-4326.

9. Pereira, A.; Martin, T.; Levinta, M.; Dujardin, C. Low-absorption, Multi-Layered Scintillating Material for High Resolution Real-Time X-ray Beam Analysis. J. Mater. Chem. C 2015, 3, 49544959.

10. Bunzli, J. C.; Comby, S.; Chauvin, A. S.; Vandevyver, C. New Opportunities for Lanthanide Luminescence. J. Rare Earths 2007, 25, 257-274.

11. Koenderink, A. F.; Alu, A.; Polman, A. Nanophotonics: Shrinking Light-Based Technology. Science 2015, 348, 516-521.

12. Fischer, S.; Hallermann, F.; Eichelkraut, T.; von Plessen, G.; Krämer, K. W.; Biner, D.; Steinkemper, H.; Hermle, M.; Goldschmidt, J. C. Plasmon Enhanced Upconversion Luminescence Near Gold Nanoparticles - Simulation and Analysis of the Interactions: Errata. Opt. Express 2013, 21, 1060610611. 
13. Eichelbaum, M.; Rademann, K. Plasmonic Enhancement or Energy Transfer? On the Luminescence of Gold-, Silver-, and Lanthanide-Doped Silicate Glasses and Its Potential for Light-Emitting Devices. Adv. Funct. Mater. 2009, 19, 2045-2052.

14. Derom, S.; Berthelot, A.; Pillonnet, A.; Benamara, O.; Jurdyc, A.-M.; Girard, C.; Colas des Francs, G. Metal Enhanced Fluorescence in Rare Earth Doped Plasmonic Core-Shell Nanoparticles. Nanotechnology 2013, 24, 495704.

15. Saboktakin, M.;Ye, X.;Chettiar, U. K.; Engheta, N.; Murray, C. B.;Kagan, C. R. Plasmonic Enhancement of Nanophosphor Upconversion Luminescence in Au Nanohole Arrays. ACS Nano 2013, 7, 7186-7192.

16. Pillonnet, A.; Berthelot, A.; Pereira, A.; Benamara, O.; Derom, S.; Colas des Francs, G.; Jurdyc, A.M. Coupling Distance Between $\mathrm{Eu}^{3+}$ Emitters and Ag Nanoparticles. Appl. Phys. Lett. 2012, 100, 153115.

17. Abdellaoui, N.; Pereira, A.; Berthelot, A.; Moine, B.; Pillonnet, A. Plasmonic Enhancement of Eu: $\mathrm{Y}_{2} \mathrm{O}_{3}$ Luminescence by Al Percolated Layer. Nanotechnology 2015, 26, 095701.

18. Gerard, D.; Gray, S. K. Aluminium Plasmonics. J. Phys. D: Appl. Phys. 2015, 48, 184001.

19. Lee, K.-T.; Park, J.-H.; Kwon, S. J.;Kwon, H.-K.; Kyhm, J.; Kwak, K.-W.; Jang, H. S.; Kim, S. Y.; Han, J. S.; Lee, S.-H.; Shin, D.-H.; Ko, H.; Han, I.-K.; Ju, B.-K.; Kwon, S.-H.; Ko, D.-H. Simultaneous Enhancement of Upconversion and Downshifting Luminescence via Plasmonic Structure. Nano Lett. 2015, 15, 2491-2497.

20. Wierer, J. J.; David, A.; Megens, M. M. III-nitride Photonic-Crystal Light-Emitting Diodes with High Extraction Efficiency. Nat. Photon. 2009, 3, 163-169.

21. Park, Y.; Drouard, E.; El Daif, O.; Letartre, X.; Viktorovitch, P.; Fave, A.; Kaminski, A.; Lemiti, M.; Seassal, C. Absorption Enhancement using Photonic Crystals for Silicon Thin Film Solar Cells. Opt. Express 2009, 17, 14312-14321. 
22. Noda, S.; Fujita, M.; Asano, T. Spontaneous-Emission Control by Photonic Crystals and Nanocavities. Nat. Photon. 2007, 1, 449-458.

23. Kedia, S.; Sinha, S. Influence of Photonic Crystal on Fluorescence Resonance Energy Transfer Efficiency between Laser Dyes. J. Phys. Chem. C 2015, 119, 8924-8930.

24. Ko, K.-Y.; Lee, Y. K.; Do, Y. R.; Huh, Y.-D. Structural Effect of a Two-Dimensional $\mathrm{SiO}_{2}$ Photonic Crystal Layer on Extraction Efficiency in Sputter-Deposited $\mathrm{Y}_{2} \mathrm{O}_{3}: \mathrm{Eu}^{3+}$ Thin-Film Phosphors. $J$. Appl. Phys. 2007, 102, 013509.

25. Ko, K.-Y.; Her, E.-J.; Nichols, W. T.; Lee, H.; Do, Y. R.; Ahn, J. Fabrication of 2D Photonic Crystal Assisted $\mathrm{Y}_{2} \mathrm{O}_{3}: \mathrm{Eu}^{3+}$ Thin-Film Phosphors by Direct Nano-Imprinting. Microelectron. Eng. 2011, 88, 2930-2933.

26. Zhang, J; Zhang, P; Ma, N; Dai, S; Zhang, W; Nie, Q. Enhancing Extraction Efficiency of MidInfrared Fluorescence in Chalcogenide Glass via Photonic Crystal. Optics Communication 2016, $364,18-23$.

27. Deschamps, T.; Guille, A.; Drouard, E.; Mazurczyk, R.; Orobtchouk, R.; Jamois, C.; Fave, A.; Peretti, R.; Fourmond, E.; Pereira, A.; Moine, B.; Seassal, C. Nanostructured Down-Converter Module for Photovoltaic Application. Proc. SPIE 2013, 8620, 86200G.

28. Hofmann, C. L. M.; Herter, B.; Fischer, S.; Gutmann, J.; Goldschmidt, J. C. Increased Upconversion Quantum Yield in Photonic Structures due to Local Field Enhancement and Modification of the Local Density of States - A Simulation-Based Analysis. Opt. Express 2016, 24, 14895-14914.

29. Ronda, C. R.; Justel, T.; Nikol, H. Rare Earth Phosphors Fundamentals and Applications. J. Alloys Comp. 1998, 275, 669-676.

30. Justel, T.; Nikol, H.; Ronda, C. New Developments in the Field of Luminescent Materials for Lighting and Displays. Angew. Chem. Int. Ed. 1998, 37, 3085-3103. 
31. Matsuura, D. Red, Green and Blue Up-Conversion Luminescence of Trivalent Rare Earth Ion-Doped $\mathrm{Y}_{2} \mathrm{O}_{3}$ Nanocrystals. Appl. Phys. Lett. 2002, 81, 4526.

32. Binnemans, K. Interpretation of Europium (III) spectra. Coor. Chem. Reviews 2015, 295, 1-45.

33. Ko, K.-Y.; Lee, Y. K.; Park, H. K.; Kim, Y.-C.; Do, Y. R. The variation of the enhanced photoluminescence efficiency of $\mathrm{Y}_{2} \mathrm{O}_{3}: \mathrm{Eu}^{3+}$ films with the thickness to the photonic crystal layer, Opt. Express 2008, 16, 5689-5696.

34. Boroditsky, M. ; Vrijen, R. ; Krauss, T. F. ; Coccioli, R. ; Bhat, R. ; Yablonovitch, E. Spontaneous Emission Extraction and Purcell Enhancement from Thin-Film 2-D Photonic Crystals. Journal of Lightwave Technology 1999, 17, 2096-2112.

35. Moharam, M. G; Gaylord, T. K. Rigorous Coupled-Wave Analysis of Planar-Grating Diffraction. $J$. Opt. Soc. Am. 1981, 71, 811-818.

36. Meng, X.; Gomard, G.; El Daif, O.; Drouard, E.; Orobtchouk, R.; Kaminski, A.; Fave, A.; Lemiti, M.; Abramov, A.; Roca i Cabarrocas, P.; Seassal, C. Absorbing photonic crystals for silicon thinfilm solar cells: Design, fabrication and experimental investigation, Sol. Energ. Mat. Sol. C. 2011, 95, S32-S38. 